\title{
An Investigation into the Significance of HHV-6 in Catatonia and Mental Health Disorders
}

\author{
Hypothesis HHV-6 can lead to encephalopathy, catatonia and \\ mental health disorders
}

\begin{abstract}
In this paper, the effects of all types of Human Herpes Virus (HHV-6) is reviewed in relation to encephalopathy, catatonia and mental health disorders due to recent research indicating that HHV-6 does a lot more than just causing Roseola, encephalitis and encephalopathy, and that it could be the key to the recent rise in mental health disorders.
\end{abstract}

The review suggests that the hypothesis that HHV-6 can lead to encephalopathy, catatonia and mental health disorders is true. The links between HHV-6, catatonia and mental health disorders are clear, and it seems necessary to do more research into the topic. It is possible HHV-6 plays a big role in causing catatonia among patients due to it causing encephalopathy and possibly causing and/or assisting the onset of mental health disorders, both of which are known to cause catatonia. HHV-6 is a relatively unknown pathogen to the wider population, but research is now indicating its prevalence and its effects could be behind the recent rise in mental health disorders.

Key words

HHV-6, Catatonia, Mental health disorders

Introduction

Infections with human herpesvirus 6 (HHV-6), a ß-herpesvirus of which two variant groups (A and B) are recognized, is very common, approaching $100 \%$ in seroprevalence. Primary infection with HHV-6B causes roseola infantum or exanthem subitum, a common childhood disease that resolves spontaneously. After primary infection, the virus replicates in the salivary glands and is shed in saliva, the recognized route of transmission for variant B strains; it remains latent in lymphocytes and monocytes and persists at low levels in cells and tissues.

It is not usually associated with disease in the immunocompetent, HHV-6 infection is a major cause of opportunistic viral infections in the immunosuppressed, typically AIDS patients and transplant recipients, in whom HHV-6 infection/reactivation may culminate in rejection of transplanted organs and death. In this paper, the effects of all types of HHV6 will be reviewed in relation to encephalopathy, catatonia and mental health disorders due to recent research indicating that HHV-6 does a lot more than just causing Roseola, encephalitis and encephalopathy, and that it could be the key to the recent rise in mental health disorders.
Sohan Das Student Essay

Haberdasher's Aske Boys School, Watford, UK

Cite as: Das, S. (2021) An investigation into the significance of HHV-6 in Catatonia and mental health disorders. Sushruta J Health Pol \& Opin vol 14; Issue 2: Art 6 ePub 12.6.21 do

https://doi.org/10.38192/14.2.6

$\underline{\text { Article Information }}$

Submitted 15.2.21

Revised 8.5.21

Published 12.6.21

ISSN 2732-5164 (Online)

ISSN 2732-5156 (Print)

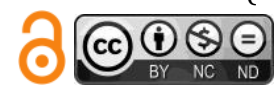

scienceOPEN.com 


\section{Why HHV-6}

HHV-6 is a very common virus. Almost $100 \%$ of people will have caught it by the age of three and won't know as it often presents with fever or diarrhoea, and its effect varies between different individuals. It often causes Roseola in young children aged 6 months to 2 years. Its effects after that age differ vastly according to the circumstances. In immunocompromised patients, particularly those receiving or recovering from stem cell transplants, reactivation of HHV-6 can cause encephalitis and/or encephalopathy. Such presentation has now also been reported in immunocompetent patients. [1] Often as a result of the onset of encephalopathy, a state of catatonia is induced. However, sometimes, in a handful of cases, a state of catatonia is seen with no other symptoms of encephalopathy, indicating no physical cause of catatonia. This is where looking into the possibility of mental health disorder-induced catatonia is introduced.

\section{The Intricacies of HHV-6}

HHV-6a and HHV-6b have been classified as two distinct herpesvirus species due to their distinctive biological properties and genome sequences. The vast majority of documented primary infections and reactivation events are due to HHV-6b. HHV-6b infects most children within the first three years of life (Roseola) and, like other herpesviruses, it establishes latency after primary infection. HHV-6b may reactivate in immunocompromised hosts, especially following allogeneic hematopoietic (stem) cell transplantation. [2]

Little is known about the epidemiology or clinical implications of HHV6a. Not much is actually known about the transmission of HHV-6. It is commonly thought that HHV-6b is transmitted through saliva, but not much is known about the transmission of HHV-6a. Theories for the transmission of HHV-6a include inhalation, and, similar to most other human herpesviruses, sexual transmission. HHV-6 is exceptional in its ability to integrate with the DNA of the host without changing the hosts DNA. Chromosomally integrated HHV-6 (ciHHV-6) is an inherited condition in which the complete HHV-6 genome is integrated into the telomere of every chromosome. The condition affects slightly less than $1 \%$ of the population in the US and UK. [3] Here, the virus lies dormant, after being passed on from mother to baby, until a secondary infection, and is then reactivated, meaning every chromosome in every single cell in the body is infected, or more accurately reinfected.

\section{HHV-6 and Catatonia}

Catatonia is an uncommon presentation of encephalopathy. Catatonia is a seemingly semiconscious state, where patients present with the following signs:

\section{Stupor (holding a rigid position without any active recognition of the environment)}

2. Catalepsy (being in one position for a long time

(similar to but not the same as stupor))

3. Waxy flexibility (allowing others to position the patient and then going into a state of catalepsy)

4. Mutism (little or no verbal response to stimuli)

5. Negativism (not responding to instructions or external stimuli)

6. Posturing (the spontaneous and active maintenance of a posture against gravity)

7. Mannerisms (odd or strange performances of a normal action)

8. Stereotypical behaviour (repetitive, meaningless movement, repeated abnormally frequently)

9. Agitation (a state of anxiety or nervous excitement-however, in catatonia, the patient will become agitated without external stimuli) 10. Grimacing (a pained/disgusted smilea grimace) 11. Echolalia (saying a phrase over and over again, which is usually meaningless, or copied from another person)

12. Echopraxia (copying another person's movements for no reason). [4]

There are three types of catatonia, stuporous, excited and malignant. [5] Each is a different presentation of catatonia.

- In stuporous catatonia, the most common one, the patient goes into a state of stupor and is oblivious or non-reactive to external stimuli. The person stays in a cataleptic state for hours, making no eye contact or noise.

- Excited catatonia is where the patient is in a state of constant agitation, with no purpose. They may experience delusions and hallucinations at the same time - it is considered one of the most dangerous mental states to be in.

- In malignant catatonia, the patient has an acute onset of fever, excitement, autonomic instability and delirium-a combination of these symptoms can be fatal. HHV-6 can cause and has been known to cause catatonia through the development of 
encephalopathy during the primary infection or reactivation.

Common causes of encephalopathy include infection, immune disorder or organ failure (liver damage, kidney failure etc). Infectious diseases known to cause encephalopathy and therefore catatonia include HIV, meningitis, all types of herpes (including HHV-6) and both hepatitis B and C. [6] HHV-6 has also been associated with encephalomyelitis/chronic fatigue syndrome, a disease characterised by severe limitations of physical activity. This is similar to the effect of HHV6 on motor function as in catatonia. $[7,8]$

Mental Health disorders and Catatonia Mental health disorders are on the rise. According to the mental health charity Mind, 1 in 4 people in the UK will experience a mental health problem each year, [9] and in England, 1 in 6 people report experiencing a common mental health problem (such as anxiety and depression) in any given week. [10] There are many different kinds of mental health disorders, common ones like depression, anxiety, obsessivecompulsive disorder, post-traumatic stress disorder and less common but more serious ones, like bipolar disorder, severe phobias, psychotic disorders for example schizophrenia, personality disorders and many more. Statistics are available on Mind's website for how common each type of disorder is. [11]

Catatonia is commonly associated with mental health disorders.

The prevalence of catatonic syndrome varies between $5.3 \%$ - 19\% in acute psychiatric wards. $[12,13]$ It is typically associated with schizophrenia, posttraumatic stress disorder, psychosis, schizoaffective disorder, as well as major depressive disorders and bipolar disorders. It is estimated that $50 \%$ of all bipolar disorder sufferers will experience catatonia, which is why it is so important that we find out why catatonia is a common presentation of such a variety of mental health disorders. The molecular hypothesis suggests that catatonic signs and symptoms in neuropsychiatric disorders are based on genetic abnormalities. One such gene has been identified as SNORD115. Patients with this gene may have vulnerability to respond to environmental stresses with psychiatric presentations including catatonia.[14]

\section{HHV-6 and Mental Health Disorders}

It was long thought that viruses don't have anything to do with mental health disorders. However, a recent study has given us reasons to doubt that. [15] In this international study, primarily led by Bhupesh Prusty, the post-mortem posterior cerebellum of sufferers of mental illness were examined for signs of both HHV-6a and HHV-6b. The mental illnesses that were studied were 50 cases of bipolar disorder (BPD), 50 cases of schizophrenia (SCZ), 15 cases of major depressive disorder (MDD), and 50 control samples. DNA qPCR, immunofluorescence and FISH studies were carried out on every sample, looking for traces of both strains of HHV-6.

The study found significantly high levels of HHV6a protein and DNA in MDD and BPD as compared to the controls. High levels of HHV-6b proteins and DNA were also found in the cases of MDD. In fact, high levels of both strains of HHV- 6 were found in the 15 cases of MDD, as well as fairly significant levels of both in BPD and SCZ. The paper also found a significant effect in terms of gender in the amount of HHV-6 DNA and proteins present, with females presenting with more HHV-6a proteins and HHV-6b proteins than the men, but gender was later found to play no effect in HHV-6 infection. In fact, none of the clinical and demographic variables they examined correlated significantly with HHV-6a or HHV6b infection, except the age of illness onset, which was higher in psychiatric patients with HHV$6 \mathrm{~b}$ protein and DNA. The study also found that HHV6 had an effect on the Purkinje cells-which are neurons in the cerebellar cortex that regulate and coordinate motor movement. The cross-sectional soma area of RBFOX2-positive Purkinje cells was assessed for all cases to make comparisons across HHV-6a/b protein/DNA status groups, and the results were as follows. For the cells with traces of HHV-6a, the cross-sectional soma area was smaller compared to non-infected Purkinje cells, and for the cells with traces of HHV-6b the cross-sectional soma area was larger as compared to non-infected cells. The reason Purkinje cells were researched into by the scientists carrying out the study was because reduced numbers of Purkinje cells, cerebellar atrophy and microstructural/metabolic abnormalities have been reported in BPD. [16]

\section{Purkinje Cell}

There is a clear link between the two finds, HHV-6a decreased the cross-sectional soma area of the Purkinje cells, and in BPD, the cross-sectional soma area of the Purkinje cells was found to be reduced. This suggests that HHV-6 could play a role in bringing about mental health disorders in infected people, especially those with ciHHV-6, where the HHV-6 would already be present in the brain from birth. The fact that it controls motor movement 
could indicate that it has a role in causing catatonia and its physical manifestations.

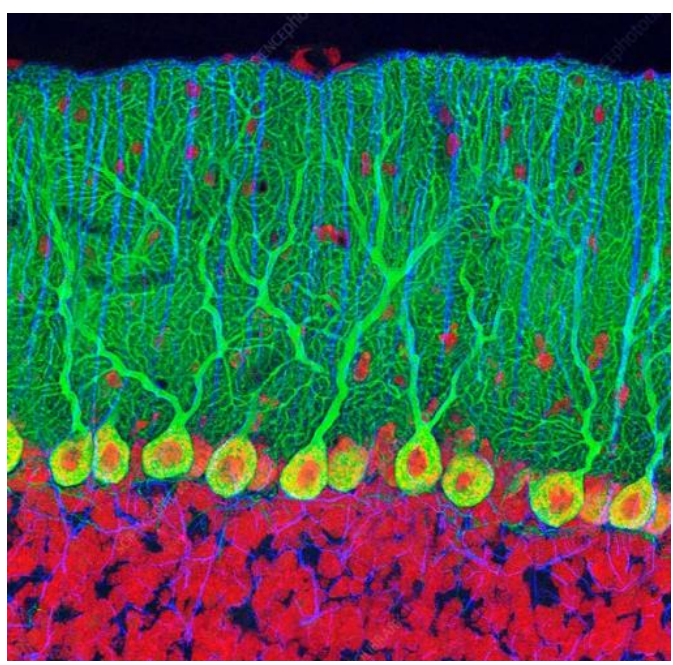

Illustration of a Purkinje cell [17]

\section{Conclusion}

The review suggests that the hypothesis that HHV-6 can lead to encephalopathy, catatonia and mental health disorders is true. The links between HHV-6, catatonia and mental health disorders are clear, and it seems necessary to do more research into the topic. It is possible HHV-6 plays a big role in causing catatonia among patients due to it causing encephalopathy and possibly causing and/or assisting the onset of mental health disorders, both of which are known to cause catatonia. HHV-6 is a relatively unknown pathogen to the wider population, but research is now indicating its prevalence and its effects could be behind the recent rise in mental health disorders.

\section{References}

1. Ongrádi J, Ablashi DV, Yoshikawa T, Stercz B, Ogata M. Roseolovirusassociated encephalitis in immunocompetent and immunocompromised individuals. J Neurovirol. 2017 Feb;23(1):1-19. doi: 10.1007/s13365-016-0473-0. Epub 2016 Aug 18.

2. Düver F, Weißbrich B, Eyrich $M$, Wölfl $M$, Schlegel PG, Wiegering V. Viral reactivations following hemopoietic stem cell transplantation in pediatric patients A single center 11-year analysis. PLoS One. 2020 Feb 4;15(2):e0228451. doi: 10.1371/journal.pone.0228451.

eCollection 2020
3. Pellett PE, Ablashi DV, Ambros PF et al: Chromosomally integrated human herpesvirus 6: questions and answers. Rev Med Virol. 2012 May;22(3):144-55. doi: 10.1002/rmv.715

4. American Psychiatric Association (2013). Diagnostic and Statistical Manual of Mental Disorders (Fifth ed.). Arlington, VA: American Psychiatric Publishing. pp. 119121. ISBN 978-0-89042-555-8.

5. M Justin Coffey, MD https://www.uptodate.com/contents/ catatonia-in-adults-epidemiologyclinicalfeatures-assessmentanddiagnosis?search=cataonia $\% 20 \mathrm{in} \% 2$ 0adults \&source=search_result \&select edTitle $=2 \sim 48 \&$ usage_type $=$ default $\& d$ isplay_rank=2

6. Davis, Charles Patrick. "Encephalopathy". MedicineNet $13 / 8 / 2018$

https://www.medicinenet.com/encep halopathy/article.htm

7. Blomberg J, Rizwan M, Böhlin-Wiener A, Elfaitouri A, Julin P, Zachrisson O, Rosén A, Gottfries CG (2019). Antibodies to Human Herpesviruses in Myalgic Encephalomyelitis/Chronic Fatigue Syndrome Patients. Front Immunol. 2019 Aug 14;10:1946. doi: 10.3389/fimmu.2019.01946. eCollection 2019.

8. Aoki R, Kobayashi N, Suzuki G, Kuratsune H, Shimada K, Oka N, Takahashi M, Yamadera W, Iwashita $M$, Tokuno S, Nibuya M, Tanichi M, Mukai Y, Mitani K, Kondo $\mathrm{K}$, Ito $\mathrm{H}$, Nakayama K Human herpesvirus 6 and 7 are biomarkers for fatigue, which distinguish between physiological fatigue and pathological fatigue. Biochem Biophys Res Commun. 2016 Sep 9;478(1):424-430. doi: 10.1016/j.bbrc.2016.07.010. Epub 2016 Jul 7.

9. McManus, S., Meltzer, H., Brugha, T. S., Bebbington, P. E., \& Jenkins, R. (2009). Adult psychiatric morbidity in England, 2007: results of a household survey. The NHS Information Centre for health and social care.

10. McManus $S$, Bebbington $P$, Jenkins $R$, Brugha T. (eds.) (2016). Mental health and wellbeing in England: Adult psychiatric morbidity survey 2014. Leeds: NHS digital.

11. https://www.mind.org.uk/information support/types-of-mentalhealthproblems/statistics-and-facts- 
aboutmental-health/how-common-

aremental-health-problems

12. Subramaniyam BA, Muliyala KP, Hari Hara $S$, Kumar Reddi VS. Prevalence of catatonic signs and symptoms in an acute psychiatric unit from a tertiary psychiatric center in India. Asian J Psychiatr. 2019 Aug;44:13- $17 . \quad$ doi: 10.1016/j.ajp.2019.07.003. Epub 2019 Jul 5.

13. Takács R, Asztalos M, Ungvari GS, AntosikWójcińska AZ, Gazdag G. The prevalence of catatonic syndrom e in acute psychiatric wards. Psychiatr Pol. 2019 Dec 31;53(6):1251-1260.

doi: 10.12740/PP/102657. Epub 2019 Dec 31.

14. Peter-Ross EM. Molecular hypotheses to explain the shared pathways and underlying pathobiological causes in catatonia and in catatonic presentations in neuropsychiatric disorders. Med Hypotheses. 2018 Apr;113:54-64. doi: 10.1016/j.mehy.2018.02.009. Epub 2018 Feb 15.

15. Prusty BK, Gulve N, Govind S, Krueger GRF, Feichtinger J, Larcombe L, Aspinall R, Ablashi DV and Toro CT (2018) Active HHV-6 Infection of Cerebellar Purkinje Cells in Mood Disorders. Front. Microbiol. 9:1955. doi: 10.3389/fmicb.2018.01955

16. DelBello, M. P., Strakowski, S. M., Zimmerman, M. E., Hawkins, J. M., and Sax, K. W. (1999). MRI analysis of the cerebellum in bipolar disorder: a pilot study. Neuropsychopharmacology 21, 6368. doi: 10.1016/S0893-133X (99)000263

17. https://media.sciencephoto.com/image/p 3600474/800wm/P3600474-

Purkinje nerve cells in the cerebellum.jp g

Key Definitions

HHV-6:

HHV-6 is the abbreviation for human herpesvirus 6, a type of virus from the family Herpesviridae that affects all ages of people but is more likely to infect the young and the old. There are 2 types - HHV-6a and HHV-6b, both very different strains of the virus. HHV6b can cause Roseola, also called exanthema subitum and/or Sixth disease-which is characterised by a high fever, followed by a rash and then by cold-like symptoms. Both strains of HHV-6 has also been linked with causing encephalitis in immunocompromised people.
Catatonia:

Catatonia is an abnormality of movement and behaviour arising from a disturbed mental state. It may involve repetitive or purposeless activity, catalepsy, resistance to passive movement and negativism. It can arise from underlying psychiatric and general medical health disorders but is not a disorder in itself.

Mental Health Disorders:

Mental health disorders comprise a broad range of problems, with different symptoms. However, they are generally characterized by some combination of abnormal thoughts, emotions, behaviour and relationships with others. Examples are bipolar disorder, depression, obsessive compulsive disorder, personality disorders, schizophrenia, disorders due to drug abuse etc. Most of these disorders can be successfully treated over differing periods of time.

\section{Encephalitis:}

Encephalitis is an uncommon, serious disease, where the brain is inflamed or swollen, with many serious symptoms including confusion or disorientation, seizures or fits, changes in personality and behaviour, difficulty speaking, weakness or loss of movement in some parts of the body and even loss of consciousness. It is caused by viral or bacterial infection or can be of immunological origin.

Encephalopathy:

Encephalopathy is a term that means brain disease, damage, or malfunction. Encephalopathy can present with a very broad spectrum of symptoms that range from mild, such as some memory loss or subtle personality changes, to severe, such as dementia, seizures, coma, or death. In general, encephalopathy is manifested by an altered mental state that is sometimes accompanied by physical manifestations (for example, poor coordination of limb movements). 